\title{
Facial nerve preservation by posterior fossa transmeatal microdissection in total removal of acoustic tumours
}

\author{
R. W. RAND AND T. L. KURZE \\ From the Divisions of Neurological Surgery, School of Medicine, University of California, Los Angeles, and \\ University of Southern California, School of Medicine, U.S.A.
}

Intracranial preservation or reconstruction of the facial nerve during total removal of acoustic tumours has infrequently been possible heretofore, the facial nerve being preserved in only $10 \%$ to $32 \%$ of operations performed by neurosurgeons (Bucy, 1951; Dandy, 1941; Dott, 1955, 1958; Drake, 1960; Horrax, 1950; Nielsen, 1942; Pool and Pava, 1957; Revilla, 1947; Olivecrona, 1950). The need for improved surgical techniques to prevent facial palsy in the majority of cases in which a tumour has been wholly removed has been recognized for many years and was emphasized by Pool and Pava in 1957: 'The problem stemming from facial nerve involvement is of considerable importance if only because of its psychological ramifications. It may become more acute if the corneal anaesthesia should be also present.'

McKenzie and Alexander (1955) concentrated on performing total removal, including the intrameatal portion of acoustic tumours, and made no particular effort to save the facial nerve. They pointed out that it was more important to cure the younger patient than to preserve the facial nerve. It is well known that total removal of acoustic neurinomas after recurrence is extremely difficult (Ransohoff, Potanos, Boschenstein, and Pool, 1961). Facial hypoglossal nerve anastamosis was done at a later stage with satisfactory restoration of facial tone and symmetry.

The posterior fossa transmeatal approach to be described here does permit the facial nerve either to be preserved during removal of the whole of the acoustic tumour, or reconstructed with or without an intracranial nerve graft should this be necessary. In order to carry out such dissection the binocular surgical microscope is required.

This approach also provides ready access to the normal contents of the internal auditory canal for selective section of the superior or the inferior vestibular nerve without sacrificing the cochlear or the facial nerves.

\section{OPERATIVE TECHNIQUE}

The sitting posture with hypothermia to $30^{\circ} \mathrm{C}$. under general anaesthesia has been employed (Rand, 1957). The patient's head is held in a specially designed unit with four-point skull fixation above the ears and supraorbital ridges, respectively. This unit allows head flexion during the opening and the resective stages of the operation, and head extension for wound closure without tension.

A unilateral incision is started below the tip of the mastoid process passing above the attachment of the suboccipital muscles to end in a vertical limb in the midcervical area (McKenzie and Alexander, 1955). A strip of fascia and muscle is left attached to the superior nuchal line to provide for tight closure. A generous unilateral craniectomy in large tumours includes the bone superior to the lateral sinus, the ipsilateral side of the foramen magnum, and the region anterolateral to the mastoid cells. A paramedian incision may be used and the wall of the foramen magnum may be left intact when the cerebello-pontine angle tumour is small (Bucy, 1951). Especially with small tumours the half axial prone posture may be useful (Mount, 1945). This allows the cerebellum to fall away from the tumour by gravity and with mild retraction.

The dura is opened in a cruciate design and its flaps are sutured to the wound edges or it may be opened around the edge of the craniectomy leaving dura covering the cerebellum. In the sitting posture, the cerebellar hemisphere hangs down in a loose fashion due to shrinkage from the use of hypothermia, and intravenous urea or mannitol, which is started slowly at the beginning of the procedure. The systolic blood pressure is not allowed to fall below $100 \mathrm{~mm}$. of mercury. Transfusion is commenced at the beginning of the operation, allowing volume-for-volume replacement.

In order to lessen postoperative swelling and avoid air embolism, care must be taken not to damage the superior veins draining the cerebellum. The cerebellum is retracted gently upward without compressing the brain-stem, using a self-retaining retractor. In this way, access to the entire lateral wall of the posterior fossa and cerebellopontine angle is gained. The jugular and acoustic foramen and their contents are readily identified grossly and 
especially well with the binocular operating microscope. The lateral portion of the cerebellar hemisphere is not resected. If the acoustic tumour is especially large a portion of it may be removed to expose the edge of the porus acousticus.

Care is taken to preserve the arachnoid over the lower cranial nerves. Only a small opening is made in the cisterna magna to allow for cerebrospinal fluid drainage. Thus, any bone debris from drilling and blood which escapes suction will not get into the subarachnoid space and can be irrigated away.

The dura mater over the posterior superior wall of the porus acousticus is carefully coagulated under magnification of the surgical microscope after identifying the tumour. It is removed from the bone with sharp curettes. Drill dissection is used to eradicate the posterior and superior walls of the internal auditory canal. The cutting drill may be employed until soft tissue is approached and then diamond drills, which do not cut soft tissue, are substituted. A combination irrigation-suction instrument is used during the drilling to remove bone dust and to cool the bone. The use of cottonoid strips and pledgets in the immediate area of drill dissection must be avoided because they may be caught in the drill. The wall of the porus acousticus is removed laterally, preserving the tumour capsule, until the origin of the tumour is visualized. One can identify the superior and inferior vestibular nerves through the microscope which are usually involved to a greater or lesser extent in the origin of the tumour. With careful dissection between these nerve fibres, the cochlear nerve can be seen anteriorly and inferiorly. It may be saved in certain cases of small tumour if it is not directly involved.

In the most antero-superior portion of the internal auditory canal, the facial nerve will be found to be free of the tumour capsule. Confirmation may be obtained by direct electrical stimulation and observation of facial movements. The neurinoma is then gently teased out of the canal with curettage, suction, and cup forceps after cutting the vestibular nerves lateral to the origin of the tumour. The tumour capsule is separated from the facial nerve by continued gentle, sharp and blunt dissection under the surgical microscope: Excessive mechanical stretching will usually result in facial movements easily observed by the anaesthetist. The air cells in the petrous bone may then be sealed with bone wax, gelfoam, or muscle stamp to prevent cerebrospinal fluid otorrhoea.

Once the internal auditory canal is cleared of the tumour, attention is directed to decompressing the tumour (Cushing, 1917), if necessary, by internal curettage, suction and/or an electrosurgical loop. As the tumour mass is decreased in size, the capsule is grasped gently with cup forceps and the cleavage plane between the tumour and the brain-stem is identified. At this point, the major vessels passing from the brain-stem to the tumour can be seen and dissected from the capsule, bluntly or by using small cottonoid sponges. Only those vessels intimately supplying blood to the tumour are carefully coagulated and transected. Care is taken not to damage the anterior inferior cerebellar artery and its branches, which can also be observed under the microscope. The eighth nerve may need to be sectioned on the mesial side of small tumours. Care must be taken to $Z$ identify the facial nerve before this step is taken. The inferior pole of the tumour is thus freed from the brainstem and lower cranial nerves.

Attention is then directed superiorly, keeping the facial $\widehat{\varrho}$ nerve identified as the capsule is removed carefully from 0 it. The residual tumour is lifted from the brain-stem. With small neoplasms, one can identify and separate all vascular structures as well as the seventh nerve from the neurinoma without great difficulty (case 1). With larger tumours, the problem becomes increasingly more $\overrightarrow{\vec{F}}$ difficult, and consequently the seventh nerve may be $\stackrel{\oplus}{\rightarrow}$ sacrificed during total tumour resection, either because $\bar{C}$ of thinning and adherence to the capsule (case 2) or direct invasion by the tumour (case 3). In these instances, with $\overline{\bar{\sigma}}$ the use of microneurosurgical techniques, reconstruc- $\mathbb{\mathbb { D }}$ tion can be accomplished using 6-0 to 8-0 black silk sutures.

Following resection of the tumour, the posterior fossa $\overrightarrow{0}$ is searched carefully for bleeding points, which are $\rightarrow$ coagulated and a watertight closure of the dura is ac- $\vec{\omega}$ complished. The fascia, muscles, and scalp layers of the wound are sutured anatomically in the usual manner without tension or drainage.

\section{CASE REPORTS}

The posterior fossa transmeatal approach using microneurosurgical techniques was performed su@ $\rightarrow$ cessfully on three patients with small to large acoustic tumours.

CASE 1 The patient, a 49-year-old, right-handed house. wife, had had a roaring sound and sense of fullness it her right ear since 1961, followed by progressively de- or creasing hearing in that ear since early 1962.

The findings of the neurological examination were limited to the loss of hearing in the right ear with slight intention tremor in the outstretched hands.

Otological investigations showed a profound right sensorineural hearing loss with some recruitment in the higher frequencies. Békèsy audiometry was type IV. The caloric tests were inconclusive.

Neuroradiological studies of the skull, including planigrams, demonstrated that the right porus acousticus was not eroded. The positive contrast study of the cerebellopontine angle cistern showed a small acoustic tumour $1.8 \mathrm{~cm}$. in diameter on the right side (Fig. 1).

At operation on 1 May 1964, it was possible to dissect the tumour from the right porus acousticus in the manner described, preserving the seventh nerve throughout its entire distance (Fig. 2). The tumour apparently arose from the inferior vestibular nerve. The anterior inferior cerebellar artery was identified and cleared from the capsule except at one small point where it densely adhered. The capsule was left at this point to avoid damage to the artery. It was necessary to cut the proximal end of the $\mathrm{N}$ vestibular and cochlear divisions of the acoustic nerve to deliver the tumour from the brain-stem.

The patient's postoperative course was uneventful, although she developed a partial facial palsy which

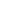




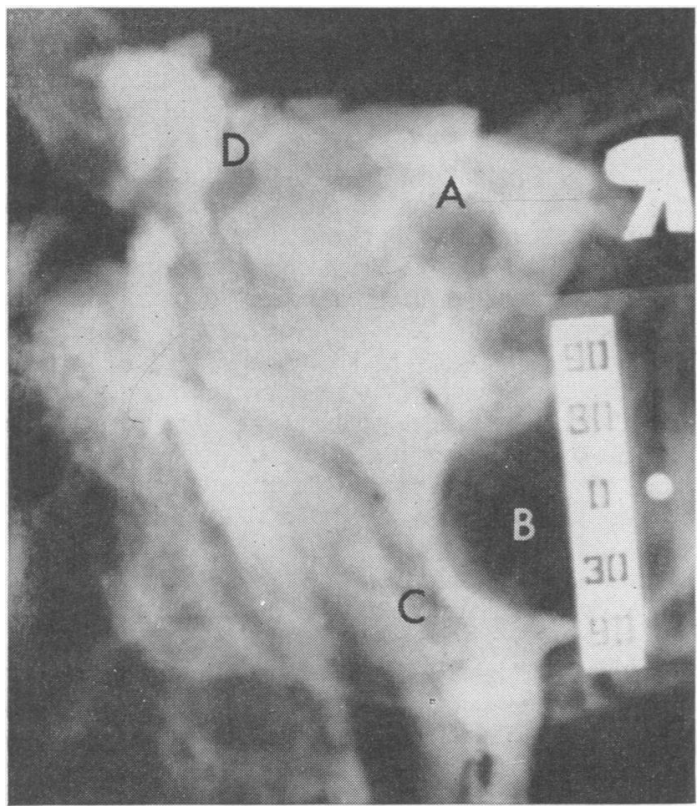

FIG. 1. Radiograph of positive contrast study of right cerebellopontine angle in case 1: (A) small acoustic tumour defect; (B) cerebellar tonsil defect; (C) vertebral and posterior inferior cerebellar arteries; (D) trigeminal nerve defect.

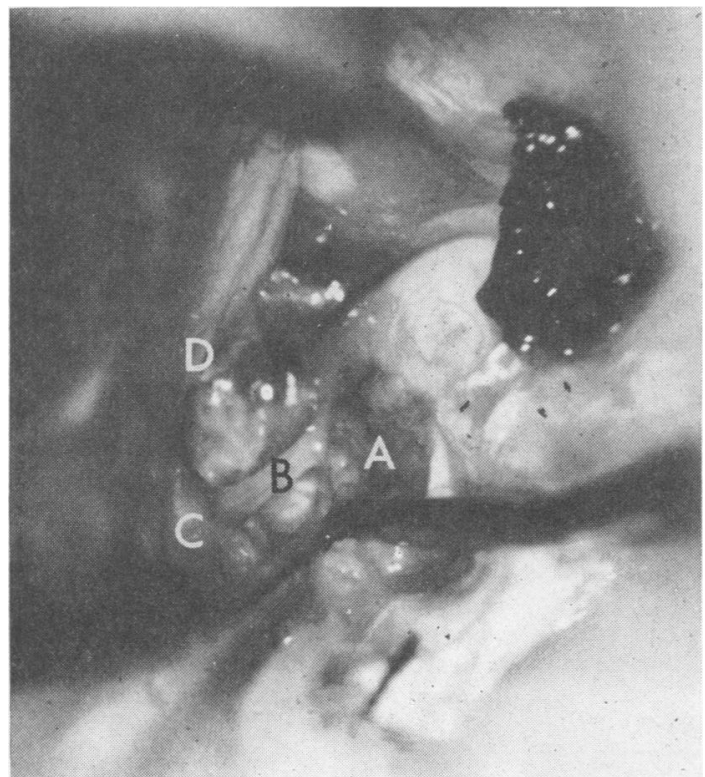

FIG. 2. Photograph through surgical microscope after resection of acoustic tumour in case 1: (A) residual neurinoma within porus acousticus removed after further unroofing of the canal by a diamond burr; (B) facial nerve intact; (C) anterior-inferior cerebellar artery; (D) brainstem and cerebellum. gradually became complete within 24 hours. Four months after surgery partial facial muscle function had returned and was nearly normal seven months after operation.

CASE 2 The patient, a 41-year-old, right-handed woman, had complaints of pulsations in the right ear, followed by tinnitus and mild deafness since 1963 . The symptoms continued in addition to her developing some numbness in the right side of her teeth and tongue.

Neurological examination demonstrated a mild variable internal strabismus without specific muscle paresis, mild horizontal nystagmus on gaze to right or left, mild hypaesthesia, and hypalgesia of the entire right side of the face, decrease of right corneal response, some weakness of the left side of the face at rest and decreased hearing on the right side. Mild unsteadiness on heel to shin testing on the right lower extremity was present.

The otological studies demonstrated sensori-neural hearing loss on the right with recruitment at high frequencies only. Békèsy audiometry was type IV. The caloric tests were equivocal.

Neuroradiological studies of the skull, including planigrams, demonstrated that the right porus acousticus was eroded. A cerebellopontine angle positive contrast

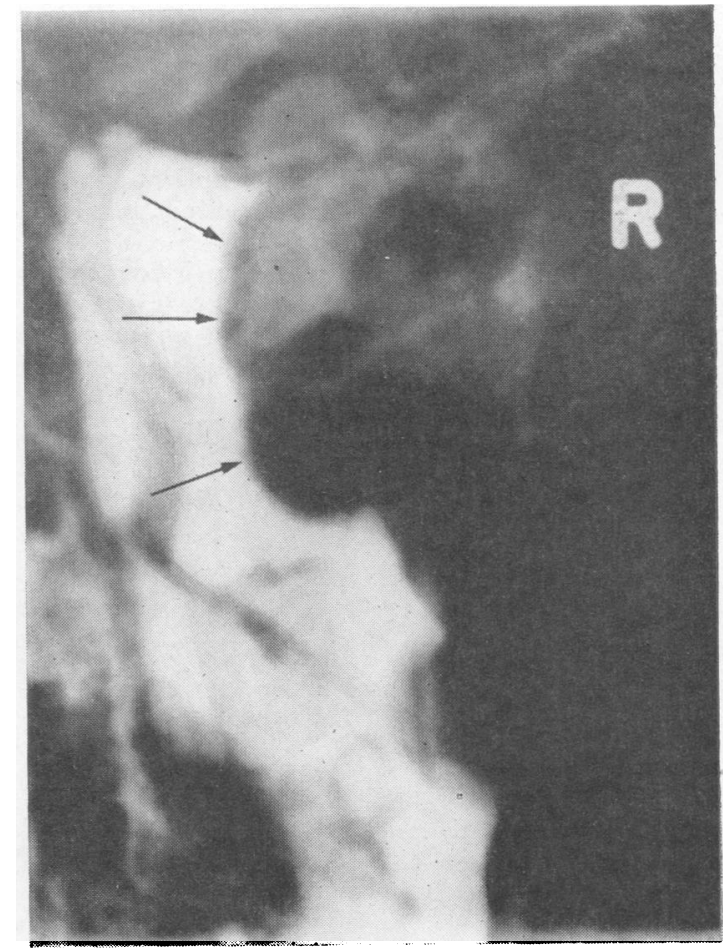

FIG. 3. Photograph of positive contrast study of right cerebellopontine angle with defect due to acoustic tumour in case 2 (arrow). Note enlarged porus acousticus in centre area of defect. 


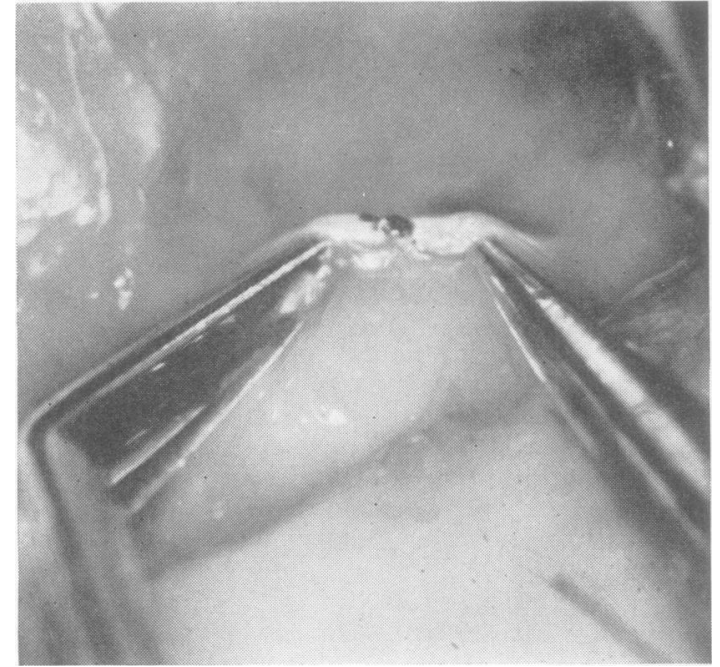

FIG. 4. Reconstruction of facial nerve by direct suture as seen through surgical microscope in case 2.

myelogram showed a large tumour $3.5 \times 2 \mathrm{~cm}$. (Fig. 3), confirming the clinical diagnosis.

At operation on 18 May 1964 the tumour was found to be massive, extending through the right side of the incisura compressing the brain-stem. It arose primarily from the inferior vestibular nerve. The seventh nerve was traced from the porus acousticus into the capsule of the tumour and from the brain-stem into the capsule where it was thinned out and severed as the neoplasm was being totally removed. The proximal and distal ends were identified and sutured with 6-0 silk (Fig. 4).

Postoperatively a tarsorrhaphy was carried out because of the corneal hypalgesia and the complete seventh nerve palsy on the right. Other neurological symptoms disappeared. The facial palsy remained complete at the eight months postoperative follow-up. Physiotherapy and $\bar{z}$ galvanic stimulation of the muscles is being continued. If facial function does not begin to return after one year a facial-hypoglossal nerve anastamosis will be performed.

CASE 3 The patient was a 40 -year-old pharmacist who, $D$ 10 years previously, had several illnesses apparently due to multiple sclerosis. The symptoms at that time involved $\stackrel{\text { ? }}{\text {. }}$ primarily the spinal cord systems and optic nerves. In 1961 he developed a progressive loss of hearing in the left . ear superimposed upon the sequelae of his multiple $\overrightarrow{\vec{F}}$ sclerosis.

Neurological examination demonstrated mild cortico- $\bar{O}$ spinal and cerebellar pathway findings, which were 흠 thought to be the result of previous disease. These con- $\overline{\bar{c}}$. sisted of a somewhat spastic-ataxic gait with hyperactive $\vec{\nabla}$ reflexes. In addition, there was loss of hearing on the left side.

Audiological studies showed a sensori-neural loss on $\vec{\theta}$ the left with recruitment. Békèsy audiometry was type II. Caloric responses were abnormal on the left.

Neuroradiological findings revealed an erosion of the left porus acousticus and a moderate sized left acoustic tumour, $3 \times 2.5 \mathrm{~cm}$. in diameter, shown in the cerebellopontine angle positive contrast study.

Operation on 24 July 1964 started with dissection in the porus acousticus. The seventh nerve was freed from $\dot{\omega}$ the tumour which arose from the vestibular nerve. The $\overrightarrow{-}$ nerve was firmly adherent to the anterior inferior surface 0 of the tumour. Eventually as resection of the tumour was carried further it was found that this portion of the faciad nerve was invaded by the neoplasm. Consequently, this segment of nerve was resected and direct anastomosig was performed after total tumour removal was cont pleted.

The patient required a tracheostomy in the third post of operative day, due to tracheo-bronchial infection and pneumonitis; this cleared under appropriate antibiotics and the site healed in a normal fashion. Tarsorrhaphy was used because of the facial palsy and decreased sensa-
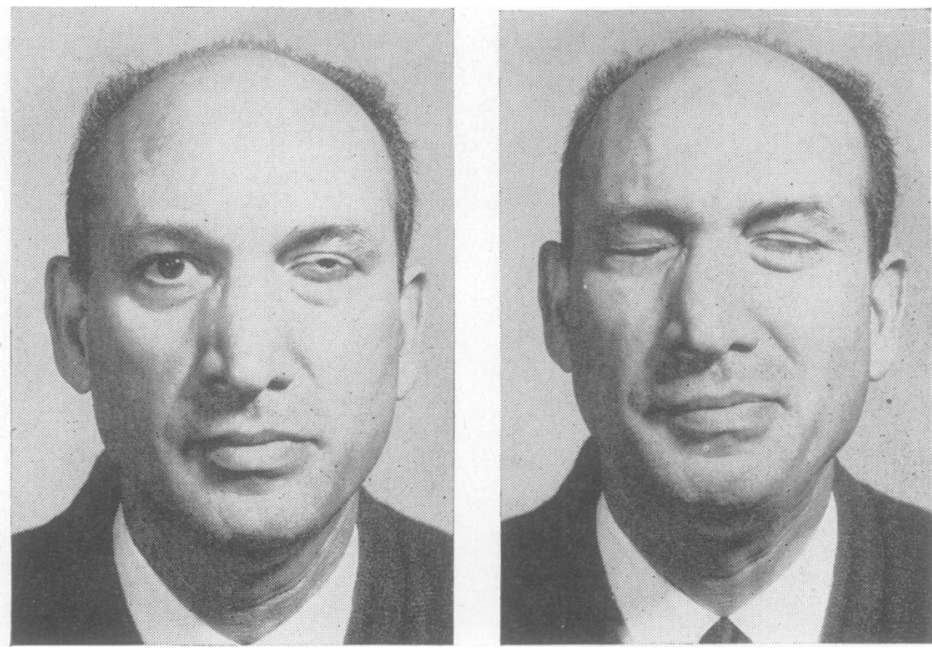

FIG. 5 Photographs of facial expressions seven months after total removal of acoustic tumour and primary intracranial facial nerve anastomosis (case 3). The tarsorrhaphy will be released when additional strength returns to the obicularis oculae muscle. 
tion in the cornea on the left side. Return of facial nerve function was first evident in the cheek five months after operation and has continued to improve about the eye and lower face.

\section{DISCUSSION}

Improved and expanded otological tests make possible earlier diagnosis of acoustic tumours as shown by a recently published monograph edited by House (1964). Pure tone loss patterns and Hallpike caloric tests were abnormal in slightly over $90 \%$ of these cases. Speech discrimination was abnormal in $69 \%$. Békèsy audiometry was type III or IV in $70 \%$. Recruitment was absent or partial in $81 \%$ and short increment sensitivity index (SISI test) scores were low in $70 \%$.

The posterior fossa cerebellopontine angle cistern positive contrast myelographic techniques perfected by Scanlan (1964) and demonstrated in Figures 1 and 3 were diagnostic of acoustic tumour in $95 \%$ of this series. The only negative study was in an intracanalicular acoustic tumour. Even these may be detected now with additional special views of the cerebellopontine angle cistern and the internal auditory canal.

An acoustic tumour arising from the internal auditory canal can be approached from three directions: (1) the subtemporal extradural middle fossa, (2) translabyrinthine, and (3) the transmeatal posterior fossa described here.

Kurze (1958) was the first to advocate the middle fossa extradural approach and a number of acoustic tumours were totally removed in this manner (House, 1964; Kurze and Doyle, 1962). The standard SpillerFrazier craniectomy was performed and the greater superficial petrossal nerve identified using extradural dissection. The canal of this nerve was unroofed by diamond burr dissection to the geniculate ganglia of the facial nerve. The seventh nerve was further unroofed by drill dissection between the labyrinth and the cochlea into the internal auditory canal. The major disadvantages to this approach are (1) the tedious and precarious diamond burr bone dissection to expose the facial nerve; (2) the limited field of exposure of the tumour even with removal of the labyrinth; and (3) the interposition of the tumour between the brain-stem vessels and the surgical field.

The translabyrinthine approach originally postulated by Panse (1904) was perfected by House (1964). A partial mastoidectomy and total labyrinthectomy are carried out, exposing the internal auditory canal in the limited area between the jugular bulb, the sigmoid sinus, the fallopian canal of the facial nerve, and the superior petrossal sinus. The cerebellopontine angle is then further exposed by partially opening the dura mater lining the posterior fossa adjacent to the cerebellum. The tumour is removed piecemeal.

Forty-four per cent of tumour resections by this route were necessarily partial because of the size of the tumour, intimate attachment of the neoplasm to the anterior inferior cerebellar artery and/or brain-stem, and haemorrhage. However, of the 23 tumours totally removed no residual damage of the facial nerve function was observed in $56.5 \%$. Mild to severe weakness was present in the remaining 10 cases. It is apparent that these results of facial nerve preservation represent a distinct improvement compared with most past neurosurgical efforts (Nielsen, 1942). However, a significant number of these benign and curable tumours were not removed totally by this technique.

The major disadvantages of this approach to the internal auditory canal are similar to the middle fossa exposure and include (1) the limited field of action, (2) the production of total hearing loss before the tumour is directly identified, (3) inability to observe and dissect directly the tumour capsule from the brain-stem and its vessels, especially the anterior inferior cerebellar artery, and (4) increased risk of cerebrospinal fluid otorrhoea.

A technique for total removal of an acoustic tumour was developed by Dandy in 1925 and has incontestable advantages, not available by the other methods of approach. The unilateral posterior fossa suboccipital craniectomy was employed. Unroofing of the porus acousticus was advocated to ensure total removal of the acoustic tumour within the canal. Preservation of the seventh nerve was limited to approximately $10 \%$ in his series.

From the experiences with the subtemporal middle fossa extradural approach, it was found that the facial nerve was most free from the acoustic tumour capsule in the lateral portion of the internal auditory canal. And we have found by extensively unroofing the porous acousticus via the posterior fossa, using drill dissection under the binocular surgical microscope, that the facial nerve can be left undisturbed in the internal auditory canal while the intrameatal portion of the acoustic tumour is being resected. It is not necessary to open the labyrinth. The remaining tumour is reduced in size by a Cushing type internal decompression (Cushing, 1917) after this initial step, and then the capsule is microdissected from the brain-stem, its vessels and the intracranial portion of the seventh nerve. With this approach, in contrast to the aforementioned, the entire dissection of the tumour can be done under direct vision and only those small vessels involving the capsule are sacrificed. The anterior 
inferior cerebellar artery and lower cranial nerves are readily identified and preserved without interposition of the tumour to obscure vision.

In the past, the lateral portion of the cerebellar hemisphere was often resected, but this is no longer necessary with the use of intravenous urea, mannitol, and drainage of the cerebrospinal fluid. The single disadvantage of this technique is cerebellar retraction which is relatively unnecessary in the translabyrinthine and middle fossa approaches to the internal auditory canal.

The advantages of the posterior fossa transmeatal technique over the extradural middle fossa and translabyrinthine approaches may be summarized as follows: (1) a wide field of action; (2) immediate and direct visualization of the anterior inferior cerebellar artery; (3) identification of the acoustic tumour before risk of damage of the facial nerve, the labyrinth, and cochlear systems; (4) dissection of the tumour is always under direct vision; and (5) direct anastomosis or reconstruction with nerve graft of the facial nerve is permitted when necessary.

\section{SUMMARY}

The principles of preservation or reconstruction of the facial nerve with microneurosurgical techniques during total removal of variously sized acoustic tumours through the posterior fossa approach are described.

Three consectuve cases of acoustic tumour are presented in which the facial nerve was preserved in one and reconstructed in two after total removal. Facial muscle function has partly returned in one patient following anastomosis.

This technique appears to incorporate all of the advantages of previously described techniques for acoustic tumour resection and avoids their disadvantages.

\section{ADDENDUM}

Since the commencement of our work and our review of the literature, the article by Rougerie and Guyot (1964) has come to our attention. The surgical microscope was not employed and partial cerebellar resection was apparently carried out. It is our experience that the difficulties Drs. Rougerie and $\bar{Z}$ Guyot had in locating the landmarks and the facial $\stackrel{\mathbb{\perp}}{=}$ nerve within the internal auditory canal do noto arise because of the magnification and visibility provided by microdissection.

Since submission of our manuscript, three additional cases of acoustic neurinoma have been oper-s ated upon by us with complete preservation of the facial nerve in each instance.

\section{REFERENCES}

Bucy, P. C. (1951). Surgical treatment of acoustic tumours. J. Neuro- $\frac{\overline{\frac{O}{\omega}}}{\bar{c}}$ surg., 8, 547-555.

Cushing, H. (1917). Tumors of the Nervus Acusticus and the Syn- $\overparen{\mathbb{D}}$ drome of the Cerebellopontile Angle. Saunders, Philadelphia.

Dandy, W. E. (1925). An operation for total removal of cerebellopontine (acoustic) tumours. Surg. Gynec. Obstet., 41, 129-148.

(1941). Results of removal of acoustic tumours by the unilateral $\vec{\sigma}$ approach. Arch. Surg., 42, 1026-1033. Dott, N. M. (1955). Discussion: Total removal of acoustic nerve $\overrightarrow{\vec{\omega}}$
tumors. A.M.A. Arch. Neurol. Psychiat., 74, 454-455.

(1958). Facial paralysis-restitution by extra-petrous nerve graft. Proc. roy. Soc. Med., 51, 900-902.

Drake, C. G. (1960). Acoustic neuroma. Repair of facial nerve with autogenous graft. J. Neurosurg., 17, 836-842.

Horrax, G. (1950). A comparison of results after intracapsular enuclea- $\infty$ tion and total extirpation of acoustic tumours. J. Neurof it Neurosurg. Psychiat., 13, 268-70.

House, W. F. (1964). Transtemporal bone microsurgical removal acoustic neuromas. Arch. Otolaryng., 80, 599-676.

Kurze, T. (1958). Unpublished data.

Kurze, T., and Doyle, J. B. Jr. (1962). Extradural intracranial (midd fossa) approach to the internal auditory canal. J. Neurosur 19, 1033-1037.

McKenzie, K. G., and Alexander, E. (1955). Acoustic neuromg. Clin. Neurosurg., 2, 21-36.

Mount, L. A. (1945). The lateral position for operations in the cere bellopontine angle. J. Neurosurg., 2, 460-461.

Nielsen, A. (1942). Acoustic tumours, with special reference to enț results and sparing of the facial nerve. Ann. Surg., 115, 849-863. ज

Olivecrona, H. (1950). Analysis of results of complete and partial removal of acoustic neuromas. J. Neurol. Neurosurg. Psychiat., 13, 271-2.

Panse, R. (1904). Klinische und pathologische Mitteilungen. IV. Ein Glioms des Akustikus. Arch. Ohrenheik., 61, 251-255.

Pool, J. L., and Pava, A. A. (1957). The Early Diagnosis and Treatment of Acoustic Nerve Tumors. Thomas, Springfield, Illinois.

Rand, R. W. (1957). Hypothermia anesthesia in the sitting position. Report of two cases of acoustic neurinoma. J. Neurosurg., $\overrightarrow{\overrightarrow{7}}$ 14, 648-654.

Ransohoff, J., Potanos, J., Boschenstein, F., and Pool, J. L. (1961). Total removal of recurrent acoustic tumours. Ibid., 18, 804-810.

Revilla, A. G. (1947). Neurinomas of the cerebellopontile recess. A clinical study of 160 cases including operative mortality and end results. Bull. Johns Hopk. Hosp., 80, 254-296.

Rougerie, J., and Guyot, J. F. (1964). Essai de conservation du nerf facial dans l'ablation des neurinomes de l'angle ponto-cérébelleux. Neurochirg., 10, 13-21.

Scanlan, R. L. (1964). Positive contrast medium (iophendylate) in diagnosis of acoustic neuroma. Arch. Otolaryng., 80, 698-706. 